\section{Ginecomastia Transitória Não Puberal: Relato de Três Casos}

\section{RESUMO}

Os autores relatam os casos de 3 meninos com ginecomastia pré-puberal, de etiologias incomuns. No primeiro caso, ginecomastia bilateral teve início aos 5 anos e 10 meses de idade e fol relacionada com realimentação após doença celíaca. No segundo caso, ginecomastia unilateral foi constatada aos 8 anos e 5 meses de idade, após a introdução de análogo do $\mathrm{GnRH}$, utilizado para tratamento de puberdade precoce verdadeira idiopática diagnosticada aos 5 anos. No terceiro caso, ginecomastia bilateral surgiu aos 5 anos e 9 meses e fol associada à contaminação com estrógeno de origem não estabelecida. Nos três casos, a evolução clínica foi benigna com regressão da ginecomastia. A apresentação dos casos confirma que, nesta faixa etária, a ginecomastia, embora rara, apresenta com freqüência dificuldades diagnósticas em relação ao processo desencadeante. (Arq Bras Endocrinol Metab 1999;43/5: 389-392)

Unitermos: Ginecomastia; Ginecomastia pré-puberal; Ginecomastia por realimentação; Análogo do GnRH

\begin{abstract}
We report the case of three boys with prepubertal gynecomastia, of uncommon etiology. In the first case, bilateral gynecomastia began at the age of 5 years and 10 months and was associated with nutritional disturbance secundary to celiac disease. In the second case, unilateral gynecomastia was detected at the age of 8 years and 5 months after the introduction of a GnRH analogue for treatment of idiopathic true precocius puberty diagnosed at 5 years of age. In the third case, bilateral gynecomastia began at the age of 5 years and 9 months and was associated to estrogenic contaminant of non-identified source. In all of the cases, the clinical evolution was unremarkable with regression of the gynecomastia. The presentation of the cases confirms that gynecomastia in this age, although rare, frequently presents diagnostic difficulties in relation to the trigger off process. (Arq Bras Endocrinol Metab 1999;43/5: 389-392)
\end{abstract}

Keywords: Gynecomastia; Prepubertal gynecomastia; Refeeding gynecomastia; GnRH analogue

$\mathbf{N}$ A CLÍNICA ENDOCRINOLÓGICA É FREQÜENTE o achado de ginecomastia puberal, que ocorre mais freqüientemente nos estágios III e IV da puberdade, tem etiologia não completamente esclarecida e é usualmente transitória. Enquanto a prevalência de ginecomastia na população adulta varia de 32 a $65 \%(1,2)$ e, na puberal oscila ao redor de $50 \%(3)$, a ginecomastia pré-puberal é evento incomum. A literatura de 1919 a 1979 relata 41 casos, 11 deles com idade até 5 anos, 27 dos 6 aos 10 e 3 dos 11 aos 13 (4). Descamps e colaboradores, em 1985, relacionaram outros 16 pacientes com ginecomastia na infância (5). Casos isolados foram descritos posteriormente (6-8).

\section{apresentação de caso}

\author{
Miriam C. Oliveiva \\ Ana Paula Weidlich \\ Cristina B. Pizarro
}

\author{
Disciplina de Endocrinologia, \\ Fundação Faculdade Federal de \\ Ciências Médicas/Santa Casa de \\ Misericórdia de Porto Alegre, RS.
}


Assim como em outras faixas etárias, a ginecomastia pré-puberal parece ser o produto do aumento absoluto ou relativo dos níveis de estrógeno. Na maioria das vezes, o estradiol é alto em função do aumento do substrato (andrógenos) (9), em geral secundário a tumores da supra-renal ou testículos, ou deficiência enzimática na biossíntese de esteróides supra-renais.

É objetivo dos autores acrescentar à literatura três novos casos de ginecomastia pré-puberal, de início entre os 4 e os 6 anos de idade, todos de caráter transitório e hipóteses etiológicas incomuns.

\section{RELATO DOS CASOS}

Caso 1 - Menino com 6 anos e I mês foi encaminhado por atraso de crescimento e aumento de volume bilateral das mamas de início há 3 meses. Apresentava diagnóstico prévio, estabelecido $\mathrm{cm}$ outro Serviço, de doença celíaca, tendo o surgimento da ginecomastia coincidido com a introdução terapêutica de dieta sem glúten. História negativa para contato com estrógeno. Ao exame fisico, $105 \mathrm{~cm}$ e $14,5 \mathrm{~kg}$, ambas medidas abaixo do $3^{\circ} \mathrm{P}$, genitais pré-puberais (pêlos pubianos Tanner I, pênis com $5 \mathrm{~cm}$ de comprimento, testículos com $1 \mathrm{~cm}^{3}$ ), mama direita com $3 \mathrm{~cm}$ de diâmetro e mama esquerda com $2 \mathrm{~cm}$. Trazia avaliação da idade óssea compatível com a idade cronológica. A ecografia de tórax confirmou a presença de tecido glandular subareolar. A radiografia de tórax foi normal, assim como a função da tireóide e o beta-HCG. A testosterona total foi inferior a $0,1 \mathrm{ng} / \mathrm{ml}$ (valor de referência pré-puberal $<3,5 \mathrm{ng} / \mathrm{ml})$, a testosterona livre abaixo de $0,5 \mathrm{pg} / \mathrm{ml}(15-40)$ e o estradiol igual a $10,5 \mathrm{pg} / \mathrm{ml}(<10)$. Três meses após a primeira consulta, o paciente apresentava $106 \mathrm{~cm}, 15,3 \mathrm{~kg}$, regressão completa da mama esquerda e parcial da direita. O provável diagnóstico etiológico nesse paciente foi de ginecomastia por realimentação.

Caso 2 - Menino foi trazido à consulta aos 4 anos e 8 meses, com história de surgimento de pêlos pubianos há 8 meses. Apresentava na ocasião pêlos pubianos Tanner II, pênis com $7 \mathrm{~cm}$ de comprimento, bolsa escrotal pregueada, testículos com $3 \mathrm{~cm}^{3}$. O teste do $\mathrm{GnRH}$ mostrou aumento predominante da resposta do $\mathrm{LH}(0,1 \mathrm{mUI} / \mathrm{ml}$ basal, $10,7 \mathrm{mUI} / \mathrm{ml}$ no pico) em relação ao FSH $(0,6 \mathrm{mUI} / \mathrm{ml}$ basal, $5,2 \mathrm{mUI} / \mathrm{ml}$ no pico); a idade óssea (9 anos) apresentava acentuada aceleração em relação à cronológica (5 anos e 3 meses). Estabelecido clínica e laboratorialmente o diagnóstico de puberdade precoce verdadeira idiopáti$\mathrm{ca}$, aos 5 anos e 6 meses tor iniciado tratamento com acetato de ciproterona. Este medicamento foi utilizado por 2 anos e 7 meses, sendo então substituído por um agonista do GnRH (Triptorelina, Decapeptyl ${ }^{\circledR}$ ). Nesta ocasião, o paciente apresentava pêlos pubianos estágio II de Tanner, pênis com $7 \mathrm{~cm}$ de comprimento e testículos com $3 \mathrm{~cm}^{3}$, parâmetros estes que se mantinham estáveis desde o início do tratamento. Em consulta quatro meses após a introdução do análogo do $\mathrm{GnRH}$, apresentando $134 \mathrm{~cm}, 75^{\circ} \mathrm{P}$, foi observada aceleração no desenvolvimento dos genitais (pênis com $9 \mathrm{~cm}$, testículos com $8 \mathrm{~cm}^{3}$, pêlos pubianos entre estágios II e III de Tanner) e botão mamário à direita, sendo a presença de tecido mamário confirmada através da ultra-sonografia. A medicação foi suspensa por iniciativa da família, a qual solicitou reinício do acetato de ciproterona. Seis meses após, na vigência de ciproterona, constatoul-se regressão do tecido mamário. Neste caso, a ginecomastia foi associada ao uso do análogo do GnRH.

Caso 3 - Menino com 5 anos e dez meses veio à consulta por apresentar aumento de volume mamário bilateral há um mês. O responsável negava alterações anteriores dignas de nota ou uso de medicamentos. Ao exame, $110 \mathrm{~cm}, 18 \mathrm{~kg}, 25^{\circ} \mathrm{P}$ de altura e peso, genitais pré-puberais (pêlos pubianos Tanner I, pênis com $4 \mathrm{~cm}$ de comprimento, testículos com $2 \mathrm{~cm}^{3}$ ) e mamas. A ecografia mamária demonstrou presença de tecido mamário bilateral, simétrico, com aproximadamente $2 \mathrm{~cm}$ de diâmetro. Provas de função tireóidea, betaHCG e exame radiológico de tórax foram normais. Prolactina de $9 \mathrm{ng} / \mathrm{ml}$ (3-30), estradiol de $75 \mathrm{pg} / \mathrm{ml}$ $(<10)$, testosterona livre de $0,3 \mathrm{pg} / \mathrm{ml}(15-40), \mathrm{LH}$ de $0,1 \mathrm{mUI} / \mathrm{ml}(<5,6)$ e FSH de $0,5 \mathrm{mUI} / \mathrm{ml}(<2,0)$. Ecografias de abdome e testículo normais. Três meses após, foi observada redução no tamanho das mamas e, em nova consulta, quatro meses depois, observou-se redução total do tecido mamário. Nesta ocasião, nova dosagem de estradiol foi de $15 \mathrm{pg} / \mathrm{ml}$. A ginecomastia esteve provavelmente associada ao contato com estrógeno exógeno.

\section{DISCUSSĀO}

As prováveis etiologias da ginecomastia pré-puberal nos casos 1 e 2 são, ao conhecimento dos autores, não descritas na literatura. Além de tumores da supra-renal e testículos, e deficiências enzimáticas da supra-renal, como a da 1 lbeta-hidroxilase, outras causas tais como a administração de andrógenos, o aumento da atividade da aromatase e a concomitância com precocidade sexual, foram raras vezes associadas à ginecomastia 
pré-puberal (4). Está descrita também a associação com síndromes como a esclerose tuberosa (10) e Peutz-Jeghers, neste caso na vigência de tumor testicular (11). Uma das causas mais freqüentes de ginecomastia na população adulta, a dependente do uso de medicamentos, até há bem pouco não era relatada na infância (12). Recentemente, no entanto, foram descritos 22 casos de ginecomastia em meninos de 2 a 12 anos tratados com $\mathrm{GH}$, em $63 \%$ dos casos detectada nos primeiros seis meses de terapia (12). Mais recente ainda, é a descrição de ocorrência de ginecomastia na infầncia, como resultado de terapêutica com metoclopramida para o tratamento de refluxo gastroesofágico (13).

No primeiro caso aqui relatado, o mecanismo desencadeante da ginecomastia parece ter sido a realimentação pós-desnutrição secundária à doença celíaca não tratada. Esta etiologia foi inicialmente descrita em prisioneiros de guerra, mas também ocorre na reabilitação após doenças consumptivas (14), exercício excessivo e distúrbios psicológicos como na anorexia nervosa (15). Embora em adultos a supressão da função gonadal e a disfunção hepática que acompanham a desnutrição possam por si só se associar à ginecomastia, é mais freqüente o aumento do tecido mamário acompanhar a reintrodução da ingesta calórica. Nessa ocasião, a reativação do eixo hipófise-suprarenal e o consequiente aumento na produção de estrógenos e andrógenos levam à ginecomastia. Com a normalização da função hepática ocorre regressão do tecido mamário (16).

No segundo paciente, o uso do análogo do GnRH apresentol associação temporal com o surgimento da ginecomastia. É sabido que, antes do agonista interferir com a ação do GnRH endógeno no seu receptor nos gonadotrofos, tornando-os refratários, eles funcionam como agonistas. Nesta ação, induzem um aumento transitório na secreção de gonadotrofinas, o qual às vezes se manifesta clinicamente por aumento no desenvolvimento das características sexuais secundárias ou rápido período menstrual (17). Este fato justifica o uso, em alguns centros, de administração concomitante de acetato de ciproterona no primeiro mês de tratamento, para minimizar os efeitos do estímulo gonadal (17). No segundo mês de tratamento, os gonadotrofos se tornam refratários e ocorre supressão do eixo, a qual também pode se associar à ginecomastia (15). No caso apresentado não há dados sobre o início do aumento mamário. A constatação da ginecomastia pode ter ocorrido já durante sua involução, o que explicaria o maior lapso de tempo entre o início da terapêutica e o diagnóstico. Outra possibilidade seria o tratamento inadequado em termos de supressão do eixo gonadal, o que, apesar da ausência de comprovação através do teste agudo com $\mathrm{Gn} R H$, não nos parece viável, levando em conta o uso do medicamento em dose, freqüência e via habituais.

No terceiro paciente, os níveis séricos elevados de estradiol, uma vez eliminadas outras fontes internas de produção do mesmo, sugerem contaminação exógena, apesar de história de contato negativa. Sabe-se que é comum a história falhar em apontar fontes não óbvias de estrógeno (16). A elucidação da fonte pode ser mais dificil em jovens e crianças, devido à rara sensibilidade que os mesmos apresentam aos efeitos feminilizantes do estrógeno (18). Citam-se na literatura agentes como cremes de cabelo, loções para pele, cremes vaginais, contaminação industrial, e líquidos embalsamadores (19). Alimentos como leite, carne de aves ou vitela, foram implicados em epidemias de ginecomastia $(20,21)$. A redução dos níveis de estradiol em alguns meses reforça a hipótese etiológica sugerida.

Concluindo, os casos aqui expostos, todos com tecido mamário confirmado através de ultra-sonografia, apresentam em comum o caráter de transitoriedade, um comportamento esperado quando a etiologia está relacionada ao uso de drogas ou à realimentação. Fica caracterizada nessa amostra a dificuldade no estabelecimento do diagnóstico final em casos de ginecomastia pré-puberal.

\section{REFERÊNCIAS}

1. Carlson HE. Gynecomastia. N Engl J Med 1980;303:795-9.

2. Niewoehner CB. Nutall FQ. Gynecomastia in a hospitalized male population. Am J Med 1984;77:633-8.

3. Biro FM, Lucky AW, Huster GA, Morrison JA. Hormona studies and physical maturation in adolescent gynecomastia. J Pediatr 1990;116:450-5.

4. Haibach H, Rosenholtz MJ. Prepubertal gynecomastia with lobules and acini: a case report and review of the literature. Am J Clin Pathol 1983;80:252-5.

5. Descamps $H$, Chaussain $\mathrm{JL}$, Job JC. Les gynecomasties du garçon avant la puberte. Arch Pediatr 1985;42:87-9.

6. Leiberman E, Zachmann M. Familial adrenal feminization probably due to increased steroid aromatization Horm Res 1992;37:96-102.

7. Berensztein $E$, Belgorosky A, Dávila MTG. Rivarola MA Testicular steroid biosynthesis in a boy with a large cell calcifying sertoli cell tumor producing prepubertal gynecomastia. Steroids 1995:60:220-5.

8. West KW, Rescorla FJ, Scherer III LR, Grosfeld JL. Diagnosis and treatment of symptomatic breast masses in the pediatric population. J Pediatr Surg 1995;30:182-7.

9. August GP, Chandra R, Hung W. Prepubertal male gynecomastia. J Pediatr 1972;80:259-63. 
10. Cummings JL, Oppenheimer EY, Hochman HI. Tuberous sclerosis. Am J Dis Child 1978;132:1215-6.

11. Young S, Gooneratne S, Straus FH, Zeller WP, Bulun SE, Rosenthal IM. Feminizing sertoli cell tumors in boys with Peutz-Jeghers syndrome. Am J Surg Pathol 1995; 19(1):50-8.

12. Malozowsky S, Stadel BV. Prepubertal gynecomastia during growth hormone therapy. J Pediatr 1995; 126:659-61.

13. Madani S, Tolia V. Gynecomastia with metoclopramide use in pediatric patients. J Clin Gastroenterol 1997;24:79-81.

14. Glass AR. Gynecomastia. Endocrinol Metab Clin North Am 1994;23:825-37

15. Baker HWG. Reproductive effects of nontesticular illness. Endocrinol Metab Clin North Am 1998;27:831-50.

16. Leung AKC. Gynecomastia. Am Fam Physician 1989:39:215-22.

17. Kwigley CA, Pescovitz $\mathrm{OH}$. Premature thelarche and precocious puberty. In: Bardin CW, Editor. Current Therapy in Endocrinology and Metabolism. St. Louis: Mosby, 1997:7-13.
18. Wilson JD. Gynecomastia. N Engl J Med 1991;324:334-5

19. Mahoney CP. Adolescent gynecomastia. Differential diagnosis and management. Pediatr Clin North Am 1990:37:1389-404.

20. Kimball AM, Hamadeh R, Mahmood RAH, Khalfan $S$, Muhsin A. Ghabrial F, et al. Gynaecomastia among children in Bahrain. Lancet 1981;671-2.

21. Fara GM. Del Corvo $G$, Bernuzzi S, Bigatello A, Di Pietro C. Scaglioni S, et al. Epidemic of breast enlargement in an italian school. Lancet 1979;2:295-7.

\section{Endereço para correspondência:}

Miriam da Costa Oliveira

Rua Dona Mimi Moro 40

90480-050 Porto Alegre, RS

Fax: (051) 328-6761

E-mail: mco@portoweb.com.br 